\title{
Design and Research on Automatic Pipe Alignment Mechanism of Metal Thin-wall Pipe Bender Based on PLC Control
}

\author{
Cai Bin ${ }^{a}$, Hu Guolin ${ }^{b}$ \\ Jiangxi Vocational College of Mechanical \& Electrical Technology, Nanchang, 330013, China \\ 79190074@qq.com, ${ }^{a} 1025222495 @ q q . c o m$
}

Keywords: design and research of the automatic, PLC control, control mechanism of metal thin-walled pipe bender

Abstract: This paper mainly studies the enterprise production based on the actual needs with the design and research of the automatic control mechanism of metal thin-walled pipe bender based on PLC control. This automatic feeding machine has been illustrated from the structure, working principle and characteristics, etc. This automatic feeding machine has many advantages such as high efficiency, low cost, and high quality etc.

\section{Introduction}

Metal thin-walled curved pipe is hollow profiled pipe commonly used material, metal thin-wall pipe bending process because it has the advantage of light quality, strong rigidity and concentration of supply and demand, and a series of advantages, in the automotive, aerospace, household appliances and other equipment has extensive application. Existing in the market for metal thin-wall pipe bending, whether traditional bend molding process or welding process, basic use manual or semi-automatic operation mode, existing in the actual production of labor intensity, low efficiency, poor shape precision, its core is the lack of an effective school tube body automatically. At present, spring support pin is generally formed by cold extrusion process ${ }^{[1]}$. Compared with traditional cutting processing method, it can reduce energy consumption and improve work efficiency ${ }^{[2]}$. The existing automatic spring support pin feeding machine on the production line is prone to misinstall or overinstall springs due to some technical problems in the process ${ }^{[3]}$. Therefore, high defect rate and high production cost make it difficult to cope with market competition ${ }^{[4]}$. In view of the shortage of the above technology, this paper introduces a kind of structure design for spring supporting pin automatic feeding machine, which can realize fast, stable and reliable spring feeding.

\section{The General Scheme of Automatic Pipe Alignment Mechanism of Metal Thin-wall Pipe Bender}

Overall plan is demonstrated as shown in figure 1, one end of the long pipe clamping in fixed units, the other end by the school after the pipe head match of the activity, can make the pipe fixed length of tubing around the school head automatic bending made into the required shape.

PLC control flow chart of automatic pipe adjusting mechanism of metal thin-wall pipe bender 
Spring support pin automatic feeding machine for electric control principle of the process, through the transition of the loading mechanism from the vibration plate and support pin the workpiece through the vacuum, the forwarding to the loading position, in the sucker is expected at the same time equipped with vacuum pressure detector, ensure that each time the vacuum suction cup, by conveying mechanism from the loading location by vacuum, once learned three support pin, accurate placement under welding equipment $(5.3 \mathrm{~mm})$ electrode hole $\Phi$ electrode position, in the sucker is expected at the same time equipped with vacuum pressure detector, ensure every time before the vacuum, feeding can detect whether learn three support pin, if the lack of support pin, It is necessary to throw away the support pin that has been absorbed in the proper position and retake the material to ensure that there are 3 support pins on the electrode before welding the welding machine cylinder down.The flow chart of electrical control principle of spring supporting pin automatic feeding machine is shown in Fig. 2.

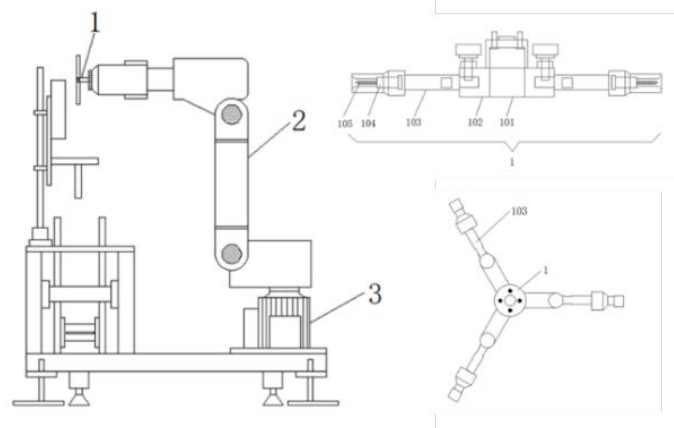

Fig. 1 General scheme diagram of automatic pipe alignment mechanism of metal thin-wall pipe bender

Automatic pipe bending pipe bender principle of the electrical control system of automatic feeding machine process roughly the following steps: first, turn on the bend pipe fittings from conveyor through vacuum suction cups and other device is transferred to the loading position waiting for fetching, Through the relevant testing on the chuck with vacuum pressure sensor, to ensure that every time when catch a suction belongs to the vacuum suction cup, by conveying mechanism from the loading location sent the vacuum suction cup can learn about three at a time of pipe body, as shown in figure 1 Each grasping Angle is 120 degrees. Accurate placement under welding equipment $(5.3 \mathrm{~mm})$ electrode hole $\Phi$ electrode position, In sucker is expected at the same time equipped with vacuum pressure detector, ensure every time before the vacuum, feeding can draw three school tube body, check if the lack of support pin, need in the appropriate position away has support pin, to take again The PLC control flow and wiring diagram of the automatic pipe bender feeding machine are shown in figure 2.
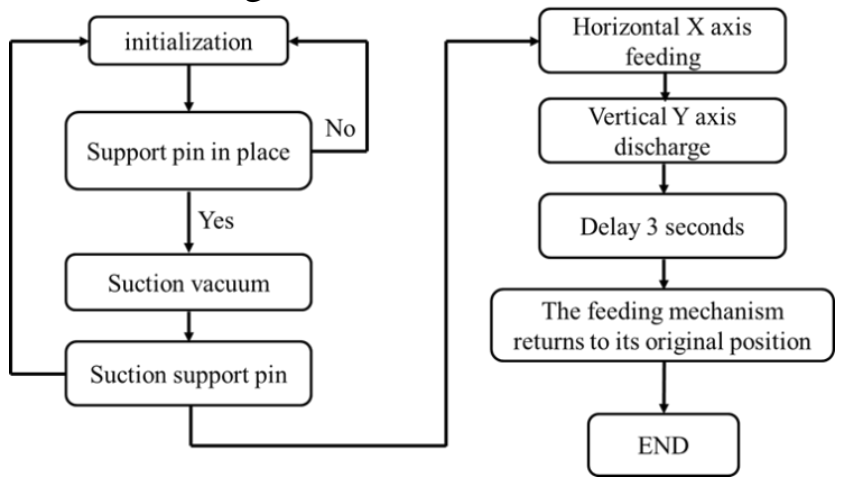

Fig.2 The PLC control flow chart of automatic pipe adjusting mechanism of metal thin-wall pipe bender 


\section{The Structure of Automatic Pipe Alignment Mechanism of Metal Thin-wall Pipe Bender}

\subsection{Electric control box}

Electric control box belongs to precision control, should close cannot reveal, electric appliance cabinet needs to install a low-voltage fluorescent lamp, easy to observe, fluorescent lamp should be made firmly. Programmable controller (PLC) control system is to use Germany's Siemens product, you can use a portable computer programs to write and to read and write operations, monitoring and maintenance, to ensure safety, touch screen with Siemens brand. The I/O interface of the logic programmable controller (PLC) shall have a certain amount of spare space for extended use, The Electric control box wiring diagram of the automatic pipe bender feeding machine are shown in figure 3.

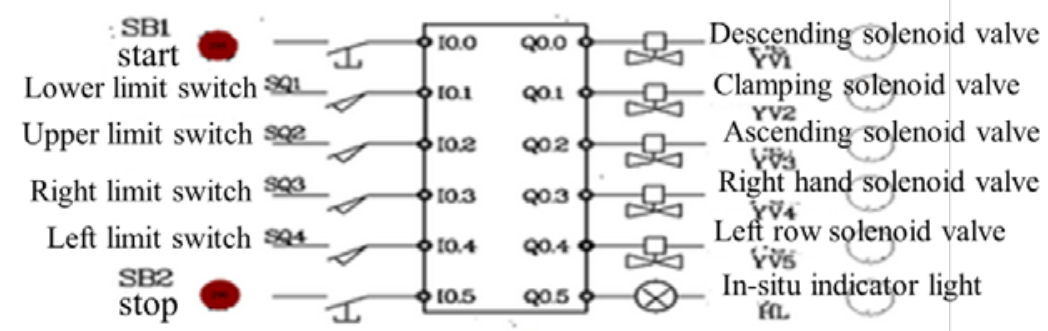

Fig.3 The Electric control box wiring diagram of the automatic pipe bender feeding machine

\subsection{Mechanical structure}

The equipment consists of vibration disk, transitional storage mechanism, feeding mechanism, vacuum detection, vacuum suction, electric and pneumatic control system, etc. Rolling institutions including mechanical structure, feeding straighteners, cut, bend forming equipment and feeding mechanism, including bending molding equipment through the center of clamping device for clamping tubes, about double bend way alone, although about two head bending forming device set up near the clamping device, can play a role positioning in the process of tube bend, but for the metal thin-wall pipe bending forming, in addition to the two ends of straight pipe, the middle parts need to be bending deformation, also means that in the process of pipe bending clamping location area is restricted, multipoint clamping not easily, if the scheme is adopted to improve the metal thinwall pipe bending processing, pipe type surface will occur fold, tube clamping The decrease of point inner diameter affects the flow rate and service life of the material. The number of support pins can be stored in the vibrator disc at one time, which is more than 3000 pieces. The function of the vibrator disc is to send the support pins out to the feeding place of the sucker plate, and arrange the support pins into a positive "vanity" arrangement. Interim storage mechanism as the main function to convey from the vibration plate to support pin artifacts by vacuum, transferred to the loading position, in the sucker is expected at the same time equipped with vacuum pressure detector, ensure every time before the vacuum, feeding can detect whether learn three support pin, if the lack of support pin, the equipment will be in the right position away has support pin, to take again. Conveying mechanism by main function from the loading location by vacuum, once learned three support pin, accurate placement under welding equipment $(5.3 \mathrm{~mm})$ electrode hole $\Phi$ electrode position, in the sucker is expected at the same time equipped with vacuum pressure detector, ensure every time before the vacuum, feeding can detect whether learn three support pin, if the lack of support pin, at the right place to throw away has support pin, to take the material again, ensure cylinder down before welding electrode welding machine has three support pin. 


\subsection{Mains input}

The power supply voltage is $380 \mathrm{hz}++10 \%, 50 \mathrm{~Hz}$ (5-wire system) or single-phase $220 \mathrm{~V}, 50 \mathrm{~Hz}$ (3-wire system). Failure can be displayed (including failure indicator). Electrical, pneumatic and other systems have safety protection measures and devices such as accidental air break and power cut to maintain the correct position of working components. The equipment shall be connected with the welding machine, and the hardware and software related to the on-line demand shall be implemented by party b. Electric control box should be closed, electrical cabinet installed a low voltage fluorescent lamp, production is firm. The programmable controller (PLC) control system adopts mitsubishi products and can use a portable computer for program reading and writing operation and maintenance monitoring. The touch screen adopts Siemens brand. The programmable controller (PLC) I/O interface should have a certain amount of spare time. 1 equipment set up the main operating box, mainly produced in welding touch screen operation, the main operating box must be set aside at least 2 root of standby signal lines, the length of the signal lines should be appropriate to wear long, signal lines at both ends of the set of online code, easy to identify. All devices in the control box shall be marked, and all cables, signal lines and terminals shall be marked with cable code, which is of moderate size and not easy to fall off and fade away. No renewal of all cables from one terminal to another is allowed. The device layout of the main control box should be reasonable, so as to make it simple and clear. The modular structure should be used as far as possible to make the disassembly and installation simple and fast.

\section{Performance Analysis}

Suppose a company has four metal thin-wall pipe bender automatic correction of pipe production line, each line need to configure an automatic machine, assuming that each device costs about 60000 yuan or so, then each class can save 1 worker, if two shifts a day, a day always total can save two workers. Assumption of minimum annual income of 40000 yuan or so, then two workers salary is about 80000 yuan, and a metal thin walled tube automatic operation of the application of pipe bending institutions and deducted from the maintenance and other related costs, we can recover the investment costs there in a year or so, at the same time, also can increase the production efficiency of the whole production line to more than $15 \%$. The enterprise has four production lines, each of which is equipped with a spring support pin automatic feeding machine. The equipment is invested in 500,000 to 600,000 yuan, saving 6 workers per production line.If the minimum income of 40000 yuan a year per person, six workers salary is about 240000 yuan, and a spring support pin the application of the automatic feeding machine operation excluding maintenance fees, can recover the cost of two to three years. At the same time, it can also increase the production efficiency to more than $20 \%$.

The global manufacturing industry will face difficulties labor costs rise, China's manufacturing has disappeared in the demographic dividend, the coming time of aging, improve the efficiency of manufacturing capacity and the reality of the demand, through the "industrial automation to replace artificial" became the trend of The Times.

\section{Conclusion}

This work mainly studies the enterprise production based on the actual needs with the design and research of the automatic control mechanism of metal thin-walled pipe bender based on PLC control. This automatic feeding machine has been illustrated from the structure, working principle and characteristics, etc. This automatic feeding machine has many advantages such as high efficiency, low cost, and high quality etc. 


\section{Acknowledgments}

This work was financially supported by the Jiangxi provincial department of education science research project Foundation (grant number GJJ161359).

\section{References}

[1] Liu Weiping, Wang Mingquan. Development and application prospect of PLC[J]. Mechanical management development, 5(2009)78-80.

[2] Zhang Fenghua, Han Baoling, Luo Qingsheng, Shi Lin, Huang Lin. Design of a new industrial stacking robot control system based on PLC[J]. Computer measurement and control, 17(2009)2191-2196.

[3] Jin Lei, Wang Jianyue. Application of configuration king and PLC in mechanical arm control system[J]. Chemical automation and instrumentation, 37(2010)103-106.

[4] Chen Yankui. The design method of PLC control system is briefly discussed[J]. China science and technology information, 20(2009)116-118. 